\title{
Development of Natural Polysaccharide-Based Nanoparticles of Berberine to Enhance Oral Bioavailability: Formulation, Optimization, Ex Vivo, and In Vivo Assessment
}

\author{
Kanchan Kohli 1,*, Ali Mujtaba ${ }^{2, *}$, Rozina Malik ${ }^{1}$, Saima Amin ${ }^{1}$, Md Sarfaraz Alam ${ }^{3}$, Abuzer Ali ${ }^{4}$, \\ Md. Abul Barkat ${ }^{5}$ and Mohammad Javed Ansari ${ }^{6}$
}

check for updates

Citation: Kohli, K.; Mujtaba, A.; Malik, R.; Amin, S.; Alam, M.S.; Ali, A.; Barkat, M.A.; Ansari, M.J. Development of Natural Polysaccharide-Based Nanoparticles of Berberine to Enhance Oral Bioavailability: Formulation, Optimization, Ex Vivo, and In Vivo Assessment. Polymers 2021, 13, 3833 https: / / doi.org/10.3390/ polym 13213833

Academic Editors: Aitana Tamayo and Maria-Dolores Veiga

Received: 6 October 2021

Accepted: 2 November 2021

Published: 5 November 2021

Publisher's Note: MDPI stays neutral with regard to jurisdictional claims in published maps and institutional affiliations.

Copyright: (c) 2021 by the authors. Licensee MDPI, Basel, Switzerland. This article is an open access article distributed under the terms and conditions of the Creative Commons Attribution (CC BY) license (https:// creativecommons.org/licenses/by/ $4.0 /)$.
1 Department of Pharmaceutics, School of Pharmaceutical Education \& Research, Jamia Hamdard, New Delhi 110062, India; ali_jh82@yahoo.com (R.M.); samin@jamiahamdard.ac.in (S.A.)

2 Departments of Pharmaceutics, Faculty of Pharmacy, Northern Border University, Rafha 73213, Saudi Arabia

3 Department of Pharmaceutics, College of Pharmacy, Jazan University, Jazan 88723, Saudi Arabia; sarfarazpharma1981@gmail.com

4 Department of Pharmacognosy, College of Pharmacy, Taif University, P.O. Box 11099, Taif 21944, Saudi Arabia; abuali@tu.edu.sa

5 Department of Pharmaceutics, College of Pharmacy, University of Hafr Al-Batin, Al Jamiah, Hafr Al-Batin 39524, Saudi Arabia; abulbarkat05@gmail.com

6 Department of Pharmaceutics, College of Pharmacy, Prince Sattam Bin Abdulaziz University, AlKharj 11942, Saudi Arabia; javedpharma@gmail.com

* Correspondence: kanchankohli55@gmail.com (K.K.); mmujtaba@nbu.edu.sa (A.M.); Tel.: +91-11-26059688 (K.K.)

Abstract: The phytogenous alkaloid berberine (BBR) has become a potential drug for the treatment of diabetes, hyperlipidemia, and cancer. However, its therapeutic potential is limited because ofpoor intestinal absorption due to its efflux by the $P$-gp expressed in the intestinal lumen. Therefore, we aimed to design and fabricate a nanoparticulate system for delivery of BBR employing naturally derived biodegradable and biocompatible polymers, mainly chitosan and alginate, to enhance the oral bioavailability of BBR. A chitosan-alginate nanoparticle system loaded with BBR (BNPs) was formulated by ionic gelation method and was optimized by employing a three-factor, three-level Box-Behnken statistical design. BNPs were characterized for various physicochemical properties, ex vivo, and in vivo evaluations. The optimized BNPs were found to be $202.2 \pm 4.9 \mathrm{~nm}$ in size, with $0.236 \pm 0.02$ of polydispersity index, zeta potential $-14.8 \pm 1.1 \mathrm{mV}$, and entrapment efficiency of $85.69 \pm 2.6 \%$. BNPs showed amorphous nature with no prominent peak in differential scanning calorimetry (DSC) investigation. Similarly, fourier-transform infrared spectroscopy (FTIR) studies did not reveal any interaction between BBR and excipients used. The drug release followed Higuchi kinetics, since these plots demonstrated the highest linearity $\left(R^{2}=0.9636\right)$, and the mechanism of release was determined to be anomalous or non-Fickian in nature. An ex-vivo gut permeation study showed that BNPs were better internalized into the cells and more highly permeated through the intestine. Furthermore, in vivo pharmacokinetic analysis in female Wistar rats showed a 4.10-fold increase in the oral bioavailability of BBR from BNPs as compared to BBR suspension. With these findings, we have gained new insight into the effective delivery of poorly soluble and permeable drugs via a chitosan-alginate nanoparticle system to improve the therapeutic performance of an oral nanomedicine.

Keywords: berberine; alginate; chitosan; nanoparticles; pharmacokinetic study

\section{Introduction}

Berberine chloride (BBR) is a naturally derived isoquinoline alkaloid found in several medicinal herbs, namely, Berberis aristata, Coptis japonica, Coptis chinensis, Hydrastis canadensis, Phellondendron amurense, and PhellondendronchinenseSchneid [1]. It has been primarily 
recommended for diarrhea and gastroenteritis, including many other biological activities, such as hypoglycemic, hypolipidemic, antiproliferative, antineoplastic, and antiarrhythmic activities [2]. It has also been found effective against diabetes, metabolic syndrome, hypertension, and polycystic ovary [3]. Thus, the drug has appeared as a therapeutic agent against multispectral activities. Due to a large number of biological activities, low cost, and low toxicity, BBR has gained more interest. BBR is prepared by chemical synthesis and available in the market as chloride and in sulfate-salt form. It is available as conventional tablets or capsules forms either alone or in combination. BBR is a yellowish crystalline powder with a bitter taste that is either odorless or has a slight odor. It is sparingly soluble in methanol and slightly soluble in ethanol and water. However, its salt forms are somewhat more soluble [4].

The wide application of BBR is, however, greatly limited by its poor intestinal absorption. The oral bioavailability of drugs depends primarily on the rate and extent of their dissolution from its dosage form. The absolute bioavailability of BBR following oral administration in rats has been reported to be below $1 \%[5,6]$. The low bioavailability of BBR is attributed to its poor aqueous solubility and dissolution, as well as its permeation by glycoprotein ( $P$-gp) through multidrug efflux pumps [7-9].

The most preferred route of drug administration is oral delivery, especially to treat chronic diseases. Many drugs fail to meet their needed therapeutic action due to their poor bioavailability, which is mainly due to poor solubility, low permeability, and/or high metabolism. Nanoparticle drug delivery systems have been extensively studied worldwide to improve the oral bioavailability of drugs with poor aqueous solubility, particularly those belonging to BCS class II and IV $[10,11]$. Biopolymers have been used as basic components of nanosystems. Specifically, chitosan and alginate have high availability, a nontoxic nature, an ease of handling, and low cost, making these polymers special and attractive choices as building blocks for nanoparticles [12].

The current research work was aimed to develop polymeric nanoparticles of BBR using alginate and chitosan as biopolymers, both of which are $P$-gp inhibitors. Further, alginate and chitosan are known to be biocompatible, biodegradable, mucoadhesive, and permeation enhancers. These properties, together with the small size of the particles, help them to cross the biological barriers and are sufficient to increase the bioavailability of BBR, which was achieved remarkably and successfully. The effect of primarily independent variables (concentration of chitosan, sodium alginate, and calcium chloride) affecting the dependent variables (particle size, polydispersity index, and zeta potential) was analyzed using a Box-Behnken design (BBD). Furthermore, the optimized formulation was characterized for various physicochemical properties inex vivoandin vivoevaluation.

\section{Materials and Methods}

\subsection{Materials}

BBR was procured from Kisalaya Herbals, Indore, India. Polymers and chitosan (medium molecular weight; degree of acetylation, 85\%) were purchased from Sigma, Cedex, France, and sodium alginate (molecular weight between 75-100 kDa) was purchased from S.D Fine Chemicals, New Delhi, India. For analysis, HPLC-grade water was used, which was taken from E-Merck India Ltd., Mumbai, India. All other chemicals and reagents, unless otherwise mentioned, were of analytical grade.

\subsection{Preparation of Berberine-Loaded Nanoparticles (BNPs)}

BNPs were prepared by cation-induced controlled gelation of sodium alginate [13]. The alginate solution was made by dissolving the polymer in distilled water and adjusted the $\mathrm{pH}$ with hydrochloric acid $(1 \mathrm{M} \mathrm{HCl})$ to $5.0-5.3$. Chitosan solution was prepared in $1 \%$ glacial acetic acid solution. For the pregelation step, $10 \mathrm{~mL}$ of calcium chloride solution was added to alginate solution in drop-by-drop with constant stirring on a magnetic stirrer at $700 \mathrm{rpm}$ for $30 \mathrm{~min}$. BBR $(5 \mathrm{mg})$ was dissolved in $1 \mathrm{~mL}$ of dimethylsulfoxide (DMSO), and it was loaded at the pregelation step. Then, $20 \mathrm{~mL}$ of chitosan solution 
was applied dropwise to the pregel step to form self-assembled nanoparticles (NPs). In order to increase overall uniformity and disperse particles, the NPs solution was stirred for 60 additional minutes, then sonicated for $15 \mathrm{~min}$, and finally homogenized for $30 \mathrm{~min}$ at 22,360 $\mathrm{g}$ forces and allowed to equilibrate for $24 \mathrm{~h}$. An ultrafiltration membrane was used, the NPs were washed with water three times to remove extra BBR and unreacted substances on the surface.

The schematic representation for the preparation of nanoparticles is as follows:

- $10 \mathrm{~mL}$ solution of calcium chloride was added dropwise to the $50 \mathrm{~mL}$ alginate solution with stirring (Pregelation step).

- At pregelation step: added BBR (5 mg was dissolved in $1 \mathrm{~mL}$ of DMSO)

- Kept it on magnetic stirrer for $30 \mathrm{~min}$

- Gelation step: added $20 \mathrm{~mL}$ of chitosan solution drop by drop

- Continue stirring for $60 \mathrm{~min}$

- $\quad$ Sonicated for $15 \mathrm{~min}$

- Finally homogenized for $30 \mathrm{~min}$ at $22,360 \mathrm{~g}$-forces

- Kept the formulation for $24 \mathrm{~h}$

Different BNP formulations were prepared and optimized using three-factor, threelevel BBD using Design Expert ${ }^{\circledR}$ software (Version 10, State-Ease Inc., Minneapolis, MN, USA). Chitosan $\left(X_{1}\right)$, sodium alginate $\left(X_{2}\right)$, and calcium chloride $\left(X_{3}\right)$ were selected as independent variables, with their low $(-1)$, medium $(0)$, and high levels $(+1)$ used for the preparation of 17 formulations. Particle size $(\mathrm{PS})\left(\mathrm{Y}_{1}\right)$, polydispersity index $(\mathrm{PDI})\left(\mathrm{Y}_{2}\right)$, and zeta potential $(\mathrm{ZP})\left(\mathrm{Y}_{3}\right)$ were chosen as dependent variables (Table 1).

Table 1. Variables and observed responses in BBD.

\begin{tabular}{|c|c|c|c|c|c|c|}
\hline \multirow[b]{2}{*}{$\begin{array}{l}\text { Formulation } \\
\text { Code }\end{array}$} & \multicolumn{3}{|c|}{ Factors Combinations at Different Levels } & \multicolumn{3}{|c|}{ Response Variables } \\
\hline & $\begin{array}{c}X_{1}=\text { Chitosan } \\
(\% \text { w/v })\end{array}$ & $\begin{array}{c}\mathrm{X}_{2}=\text { Sodium } \\
\text { Alginate }(\% \\
w / v)\end{array}$ & $\begin{array}{c}\mathrm{X}_{3}=\text { Calcium } \\
\text { Chloride }(\% \\
w / v)\end{array}$ & $\begin{array}{c}\mathrm{Y}_{1}=\text { Particle } \\
\text { Size }(\mathrm{nm})\end{array}$ & $\begin{array}{c}\mathrm{Y}_{2}= \\
\text { Polydispersity } \\
\text { Index }(\%)\end{array}$ & $\begin{array}{c}Y_{3}=\text { Zeta } \\
\text { Potential }(\mathrm{mV})\end{array}$ \\
\hline BNP1 & 0.02 & 0.3 & 0.1 & $248.3 \pm 0.02$ & $0.541 \pm 0.02$ & $-20.8 \pm 2.3$ \\
\hline BNP2 & 0.04 & 0.3 & 0.1 & $270 \pm 3.3$ & $0.414 \pm 0.05$ & $-20.9 \pm 2.3$ \\
\hline BNP3 & 0.06 & 0.3 & 0.1 & $314 \pm 6.5$ & $0.621 \pm 0.03$ & $-25.4 \pm 2.6$ \\
\hline $\mathrm{BNP} 4$ & 0.05 & 0.3 & 0.1 & $314.2 \pm 9.9$ & $0.568 \pm 0.01$ & $-13.6 \pm 3.1$ \\
\hline BNP5 & 0.08 & 0.3 & 0.1 & $341.2 \pm 2.3$ & $0.761 \pm 0.08$ & $-30.8 \pm 2.3$ \\
\hline BNP6 & 0.02 & 0.3 & 0.2 & $278.2 \pm 4.6$ & $0.496 \pm 0.06$ & $-21.8 \pm 2.7$ \\
\hline BNP7 & 0.04 & 0.3 & 0.2 & $341.2 \pm 5.7$ & $0.521 \pm 0.05$ & $-22.8 \pm 2.9$ \\
\hline BNP8 & 0.02 & 0.1 & 0.1 & $347.5 \pm 4.8$ & $0.376 \pm 0.04$ & $-27.8 \pm 1.6$ \\
\hline BNP9 & 0.02 & 0.2 & 0.1 & $246.1 \pm 5.3$ & $0.295 \pm 0.07$ & $-25.8 \pm 1.9$ \\
\hline BNP10 & 0.02 & 0.4 & 0.1 & $323.3 \pm 6.2$ & $0.504 \pm 0.03$ & $-19.8 \pm 2.1$ \\
\hline BNP11 & 0.02 & 0.5 & 0.1 & $260.4 \pm 2.9$ & $0.491 \pm 0.02$ & $-18.4 \pm 1.6$ \\
\hline BNP12 & 0.02 & 0.6 & 0.1 & $361.5 \pm 4.2$ & $0.594 \pm 0.04$ & $-30.8 \pm 1.8$ \\
\hline BNP13 & 0.10 & 0.5 & 0.1 & $290.2 \pm 8.2$ & $0.435 \pm 0.03$ & $-18.6 \pm 3.0$ \\
\hline BNP14 & 0.04 & 0.5 & 0.1 & $220.52 \pm 3.1$ & $0.311 \pm 0.01$ & $-15.1 \pm 1.6$ \\
\hline BNP15 & 0.08 & 0.5 & 0.1 & $218.6 \pm 5.1$ & $0.672 \pm 0.012$ & $-16.1 \pm 1.8$ \\
\hline BNP16 & 0.06 & 0.5 & 0.1 & $334.1 \pm 2.9$ & $0.446 \pm 0.06$ & $-11.9 \pm 2.9$ \\
\hline BNP17 & 0.02 & 0.5 & 0.1 & $253.8 \pm 6.1$ & $0.582 \pm 0.02$ & $-12.7 \pm 3.5$ \\
\hline
\end{tabular}

\subsection{HPLC Method of Analysis}

For the analytical study, an HPLC system with LC-10AT VP pump, SPD-M10A VP photodiode array detector (Shimadzu, Japan), a Rheodyne injection valve fitted with a $20 \mu \mathrm{L}$ injection loop, and CLASS-VP (Version 6.12 SP5, Tokyo, Japan) integration software were used. Baseline resolution was obtained using a Phenomenex Luna C-18 column (Phenomenex Inc., CA, USA) at $25 \pm 2{ }^{\circ} \mathrm{C}$. The mobile phase was passed via a PVDF filter of $0.45 \mu \mathrm{m}$, which was degassed before use. The flow rate was maintained constant at $0.6 \mathrm{~mL} / \mathrm{min}$, and detection was performed at a wavelength of $270 \mathrm{~nm}$ [14]. For the 
calibration plot, different concentrations of BBR $(5,7.5,12.5,17.5$, and $22.5 \mu \mathrm{g} / \mathrm{mL})$ were prepared using water as the solvent. The standard drug peak was compared with the peak of the drug sample. The HPLC calibration curve was plotted in water and plasma for determination of entrapment efficiency, drug release, and pharmacokinetics in rat plasma, respectively.

\subsection{Characterization of $B N P S$}

\subsubsection{PS, PDI, and ZP Measurement}

PS, PDI, and ZP assessment was performed through zeta sizer with DLS technique (Nano-ZS, Malvern Instruments, Malvern, UK) and analyzed by 'DTS Nano' program. The prepared BNP formulations were diluted with Milli $Q$ water and shaken vigorously to get an intensity of around $100-250 \mathrm{kV} \mathrm{S}^{-1}$. The measurement of PS, PDI, and ZP was performed for all formulations.

\subsubsection{Determination of Entrapment Efficiency (EE) and Drug-Loading (DL) Efficiency}

The EE and DL potential of the BNP formulation was measured by isolating drugloaded NPs from the aqueous phase containing non-associated BBR using ultracentrifugation (REMI Corporation, India) for $30 \mathrm{~min}$ at 20,000 rpm. 1mg of BNPs was taken and then dissolved in $1 \mathrm{~mL}$ of DMSO solution, a common solvent for BBR and alginate-chitosan complex, to dissolve them completely [15]. The above HPLC method was used to analyze the prepared solution, and the EE and DL were calculated using the given equations:

$$
\begin{aligned}
& \% \mathrm{EE}=\frac{W 1-W 2}{W 1} \times 100 \\
& \% \mathrm{DL}=\frac{W 1-W 2}{W 3} \times 100
\end{aligned}
$$

where $W_{1}=$ amount of BBR loaded in the NPs, $W_{2}=$ amount of non-entrapped BBR, and $W_{3}=$ weight of NPs

\subsection{Differential Scanning Calorimetry Analysis}

The physical state of BBR in pure-sample drug, physical mixture, and BNPs was analyzed with (Perkin-Elmer Pyres 6 DSC, Massachusetts, USA). The samples (2 mg) were weighed individually and put into an aluminum pan and then closed. As a reference standard, an empty, closed aluminum pan was placed on the alternate side. The thermal scanning was done at the rate of $10^{\circ} \mathrm{C} / \mathrm{min}$, between 40 and $300{ }^{\circ} \mathrm{C}$. The thermogram was recorded using Pyris ${ }^{\mathrm{TM}}$ software under nitrogen gas at a flow rate of $20 \mathrm{~mL} / \mathrm{min}$.

\subsection{FTIR Spectroscopy Analysis}

FTIR spectroscopic (Shimadzu, Kyoto, Japan) studies were performed with the $\mathrm{KBr}$ pellet technique. BBR and BNPs (about $5 \mathrm{mg}$ ) were separately mixed with $\mathrm{KBr}(100 \mathrm{mg})$ and were ground into a fine powder and compressed into a pellet under a hydraulic pressure at 10,000 psi. The FTIR spectra were recorded for each sample at a wave number ranging from 400 to $4000 \mathrm{~cm}^{-1}$ using Win-IR software.

\subsection{Microscopic Evaluation of BNPs}

Analysis of NPs' structural size and surface morphology was carried out using transmission electron microscopy (TEM) and scanning electron microscopy (SEM) [16]. The surface topography of optimized BNPs was observed with SEM (EVO LS 10 Zeiss, Carl Zeiss Inc., Oberkochen, Germany). The sample was spread on a slide and kept for drying. After drying, the sample was placed on carbon tape and coated with conductive gold-palladium under an argon environment in a high-vacuum evaporator using a goldsputter module. The coated samples of NPs were then scanned at an accelerating voltage of $13.52 \mathrm{kV}$, and photomicrographs were taken. Using smart SEM program tools, data analysis was performed. The surface morphology of BNPs was analyzed with Philips CM 
100 TEM. The samples were processed on copper grids, negatively stained with uranyl acetate $(2 \%)$, and air-dried before analysis. The samples were analyzed in TEM (JEOL JEM1010, Tokyo, Japan) at $100 \mathrm{kV}$ accelerated voltage, and data collection was performed on the AMT image-capture engine.

\subsection{In Vitro Drug-Release Study}

The in vitro dissolution tests were performed to analyze the drug release from BNPs formulations. The release study was carried out with a dialysis membrane bag (10 kDa molecular cutoff) in phosphate buffer $\mathrm{pH} 7.4$ with $0.5 \% v / v$ tween 80 dissolution media. The membrane was soaked overnight in the dissolution media for activation before use. A total of $10 \mathrm{mg}$ of freeze-dried BNPs was dissolved, and the equivalent quantity of BBR suspension was put in the dialysis bag and placed in dissolution media, which were stirred continuously at $150 \mathrm{rpm}$ for $24 \mathrm{~h}$ at $37 \pm 0.5^{\circ} \mathrm{C}$. The samples were withdrawn at predetermined time intervals, filtered using a $0.45 \mu \mathrm{m}$ syringe filter, and analyzed through the above-mentioned HPLC method. An equal volume of fresh dissolution media was replaced to ensure proper sink condition.

\subsection{Stability Studies}

Based on the guidelines given by ICH Q1A (R2), the stability studies of final optimized BNPs were carried out. The BNP samples were stored for 3 months in a stability chamber (TH 90S, Thermolab, Mumbai, India) at $5 \pm 2{ }^{\circ} \mathrm{C}$ and $25 \pm 2{ }^{\circ} \mathrm{C}$. The samples were analyzed for physical appearance, size analysis, drug content, and total percentage drug release at 0 , 30, 60 and 90-day intervals [17].

\subsection{Ex Vivo Gut-Permeation Study}

Ex vivo gut permeation study of BBR and BNP formulations was performed using a non-everted rat gut sac model [18,19]. The protocol for the gut permeation experiments was approved by the Institutional Animal Ethical Care Committee (IAEC) of Jamia Hamdard, India (173/CPCSEA/2012). Wistar rats of 180-200 $\mathrm{g}$ had fasted for $12 \mathrm{~h}$ before euthanasia with free access to water and were housed under standard settings. The animal was sacrificed, and the ileum part of the small intestine was cut, sliced longitudinally. A $5 \mathrm{~cm}$ long ileum part was cut and rinsed with Krebs solution to remove feces and blood.

In the gut sac, BBR and BNPs equivalent to $10 \mathrm{mg}$ of BBR were loaded. The sac was then put in the jacketed glass, having $10 \mathrm{~mL}$ of Krebs solution, which was pre-heated to $37 \pm 0.5^{\circ} \mathrm{C}$, and oxygenated via an aerator for $120 \mathrm{~min}$. The sample was removed at a specific time interval and filtered and analyzed by the above-mentioned HPLC method for the drug content. The quantity and \%drug permeation from the sac were determined and compared.

\subsection{In Vivo Pharmacokinetic Study}

Pharmacokinetic analysis was performed for optimized BNP formulations and plain BBR suspension to check the improvement of bioavailability of BBR, as per the approved experimental protocol no. 173/CPCSEA/2012 from the IAEC, Jamia Hamdard, New Delhi, India. The experiment was carried out in female Wistar rats with 180-200 g bodyweight that were kept in a cage under standard conditions ( $55 \pm 5 \% \mathrm{RH}$ and $25 \pm 2{ }^{\circ} \mathrm{C}$ ) with free access to a standard diet (Lipton feed, Mumbai, India) and drinking water. The animals were divided into three groups $(n=6)$, in which group I acts as a control and was given normal saline orally. Group II was given BBR suspension, and group III received the BNP formulation at a dose of $100 \mathrm{mg} / \mathrm{kg}$ orally. Blood samples were taken at various times after oral administration, and the plasma was separated by centrifugation at $2500 \times g$ for 20 minutes at $4{ }^{\circ} \mathrm{C}$ and kept at $-20^{\circ} \mathrm{C}$ until further examination. For bioanalysis of the sample, it is required to put the sample at room temperature, and BBR was isolated using protein precipitation technique with acetonitrile. The samples were then centrifuged, and the supernatant was collected, filtered, and analyzed by the HPLC method (Shimadzu 
LC-10 VP, Tokyo, Japan). Addins-PK Solver and Pk1Pk2 program for Microsoft Excel were used to determine the various pharmacokinetic parameters of BBR, such as maximum plasma-drug concentration $\left(C_{\max }\right)$, the time to reach maximum plasma-drug concentration $\left(\mathrm{T}_{\max }\right)$, and area under curve $\mathrm{AUC}_{0 \rightarrow \mathrm{t}}$. The relative bioavailability of the formulation was also calculated with a plain drug suspension. The pharmacokinetic results were statistically evaluated using the GraphPad Prism program (Instat 3.06, Software Inc., San Diego, CA, USA), and using the student's $t$-test, statistical differences were measured.

\section{Results and Discussion}

\subsection{Formation and Characterization of BNPs}

The chitosan-alginate nanoparticles of BBR were prepared by the ionotropic gelation method, which is a simple, rapid, and reliable method. The emulsification and organicsolvents stages were not included in the ionotropic gelation process, thereby reducing the inactivation of encapsulated drugs [20]. In the case of NPs, the addition of chitosan into the calcium-alginate pregel containing the BBR solution and the calcium alginate pregel simultaneously traps the BBR solution suspended in the medium. It is suggested that NPs can be formed by enveloping the negatively charged calcium alginate complex in a pregel state with chitosan-cationic polymer, and the pregel state is required to allow the ionic interactions between calcium, alginate, and chitosan to form NPs [21].

To statistically optimize the formulation variables, the BBD was used [22]. In order to establish the required conditions for the inclusion of the hydrophobic drug BBR into the chitosan-alginate NPs, several experiments had to be performed. A group of 17 experiments (BNP1-BNP17) was carried out for three factors at three levels each for the response surface methodology comprising BBD. In Table 1, the observed responses for all 17 formulations are shown with their independent variables of experimental runs. The amine groups of chitosan are protonated, and the carboxyl groups of alginate are ionized in aqueous solutions at a $\mathrm{pH} 5.2$, which is the most important for the formation of the polyionic complex [23]. The hydrogel is also formed at $\mathrm{pH} 5.2$ by amino-group interaction with carboxylate groups of alginate. The positively charged BBR was initially complexed with negatively charged alginate to obtain a maximum drug loading to the NPs.

The variation in PS, PDI, and ZP of the BNPs was dependent on the ratio of chitosan \%, alginate $\%$, and $\mathrm{CaCl}_{2} \%$ solution. By varying chitosan, alginate, and $\mathrm{CaCl}_{2}$ concentrations, there was a huge variation in particle size as well. The reason could be that a particular concentration of each polymer reacts with the other polymers and the rest of the amount could adhere to the surface of another polymer, hence increasing the size and causing irregularity in the shape of particles as well. The BNPs were best optimized with a stirring time of $30 \mathrm{~min}$ for pregelation and $60 \mathrm{~min}$ for gelation. Similar work was done previously, and the results obtained were almost the same [24]. All the results obtained were successfully correlated with the BBD.

The design expert software's point prediction was used to evaluate the optimum formulation of BNPs $(0.04 \% w / v$ chitosan concentration, $0.5 \% w / v$ alginate, and $0.1 \%$ $w / v$ calcium chloride) that predicted optimized process parameters to be $219.5 \mathrm{~nm}$ for PS, 0.291 for PDI, and $-15.1 \mathrm{mV}$ for ZP. The optimized BNP formulation was prepared, and the observed mean PS was $202.2 \pm 4.9 \mathrm{~nm}$, with a PDI of $0.236 \pm 0.02$ and ZP of $-14.8 \pm 1.1 \mathrm{mV}$, as shown in Figure 1a,b, respectively $(n=3)$. The predicted values of optimized formulation were compared with their experimental values for all responses, with their percentage prediction error as shown in Table 2. The results for the PS, PDI, and $\mathrm{ZP}$ were found within the limits, and the calculated (\%) prediction error ensures the reliability of the developed equations, which reveals the applicability of the RSM model [25]. 


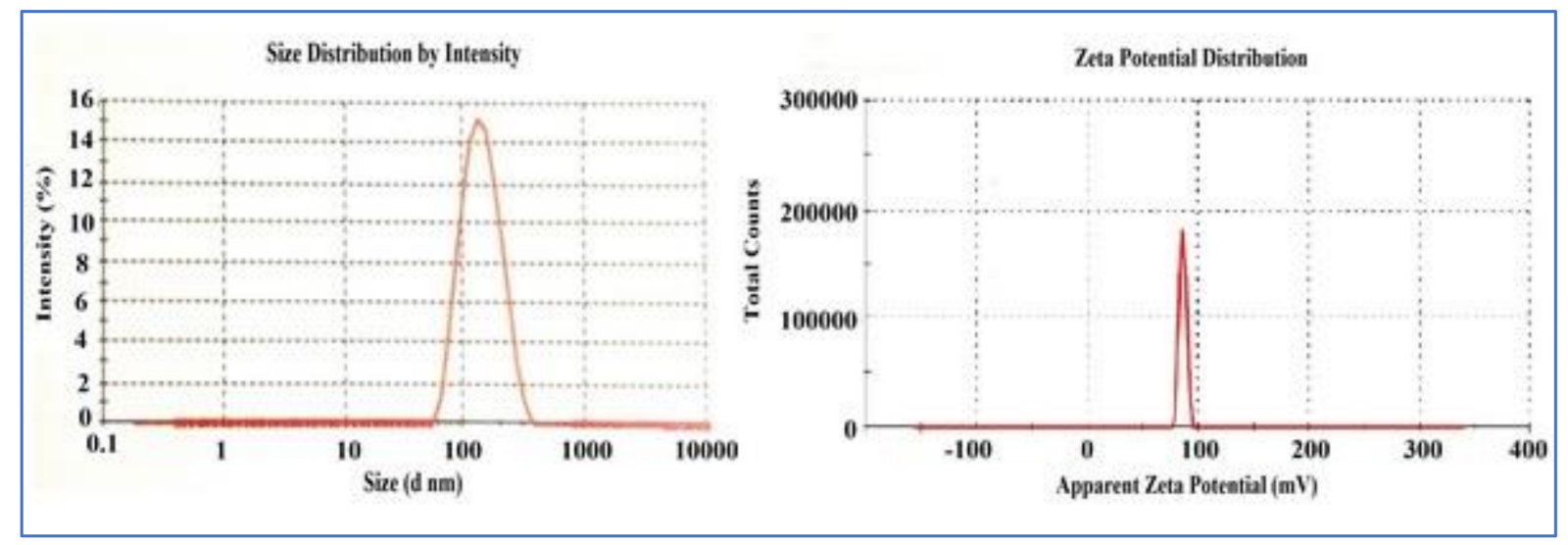

Figure 1. (a) Particle size and (b) Zeta potential of BNPs (mV).

Table 2. Composition of optimum checkpoint formulations, the predicted and experimental values of response variables, and percentage prediction error.

\begin{tabular}{|c|c|c|c|c|}
\hline $\begin{array}{l}\text { Optimized } \\
\text { BNPs } \\
\text { Formulation } \\
\text { Composition } \\
\left(\mathrm{X}_{1}: \mathrm{X}_{2}: \mathrm{X}_{3}\right)\end{array}$ & $\begin{array}{l}\text { Response } \\
\text { Variable }\end{array}$ & $\begin{array}{l}\text { Experimental } \\
\text { Value }\end{array}$ & Predicted Value & $\begin{array}{c}\text { Percentage } \\
\text { Prediction Error }\end{array}$ \\
\hline 0.04:0.5:0.1 & $\begin{array}{l}Y_{1} \\
Y_{2} \\
Y_{3}\end{array}$ & $\begin{array}{l}202.2 \\
0.236 \\
-14.8\end{array}$ & $\begin{array}{l}209.5 \\
0.241 \\
-15.1\end{array}$ & $\begin{array}{l}-2.272 \\
-2.075 \\
-1.987\end{array}$ \\
\hline
\end{tabular}

\subsection{BBR loading and Entrapment in BNPs}

The EE and DL of BBR from optimized BNP formulations were found to be $85.69 \pm 2.6 \%$ and $8.55 \pm 0.23 \%$, respectively. The entrapment of BBR in BNPs was a great challenge, and the $\mathrm{pH}$ of the alginate and chitosan solution played an important role in the EE and DL in the chitosan-alginate complex. Different $\mathrm{pH}$ was tried, but ultimately, the entrapment efficiency and loading capacity were best optimized with the $\mathrm{pH}$ of chitosan at 5.3 and that of alginate at 5.6. Similar results were also deduced by others [26]. The entrapment and loading efficiency were also dependent on the stirring time and speed of the formulation. When the stirring speed and time were increased, the entrapment and loading of the drug in BNPs were decreased, which was most probably due to the leaching of the drug due to collision among the particles and with the intact surfaces of the container for a longer time.

\subsection{DSC Analysis}

The melting point (m.p.) of BBR was found very close to the reference when analyzed on DSC and was found to be $191.58{ }^{\circ} \mathrm{C}$ [9]. In the physical mixture of BBR, chitosan, and alginate, the m.p. peak of BBR was found to be $190.51^{\circ} \mathrm{C}$, which showed no type of interaction between the excipient polymers and the drug. However, the DSC thermogram of BNPs revealed the absence of a BBR peak. The reason for the absence of a BBR peak in BNPs could be most probably due to the entrapment of BBR in solution form, or the drug could be in amorphous form. Figure 2 shows the DSC thermogram of BBR, the physical mixture of BBR with chitosan and alginate, and BPNs. 


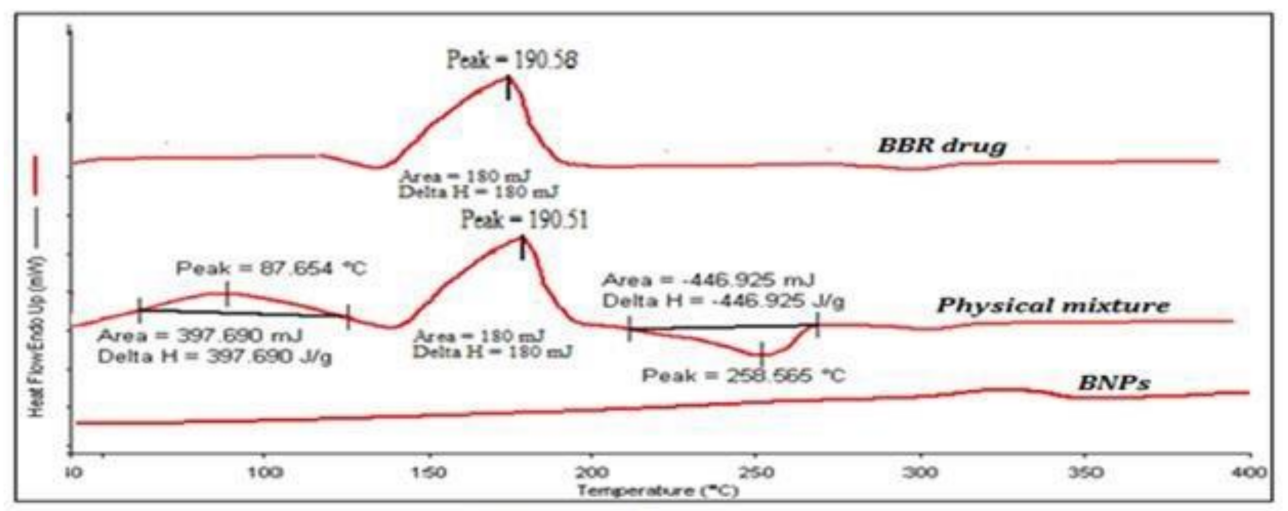

Figure 2. DSC thermograms of BBR, physical mixture, and BNPs.

\subsection{FTIR Spectroscopy Analysis}

The presence of a methoxyl group (peak at $2.844 \mathrm{~cm}^{-1}$ ) was revealed by the FTIR spectrum of BNPs, and the peak at $1.635 \mathrm{~cm}^{-1}$ is assumed to correspond to the iminium $\left(\mathrm{C}=\mathrm{N}^{+}\right)$double bond present in the molecule. In addition, the 1569 and $1506 \mathrm{~cm}^{-1}$ signals reflect the aromatic bending of $\mathrm{C}=\mathrm{C}$ and the furyl group, respectively [27]. Similar peaks are there in the BBR, which clearly support the results of DSC and reveal that there was no chemical interaction among the BBR, chitosan, and alginate in BNPs. Figure 3a,b show FTIR images of standard drug BBR and BNPs.

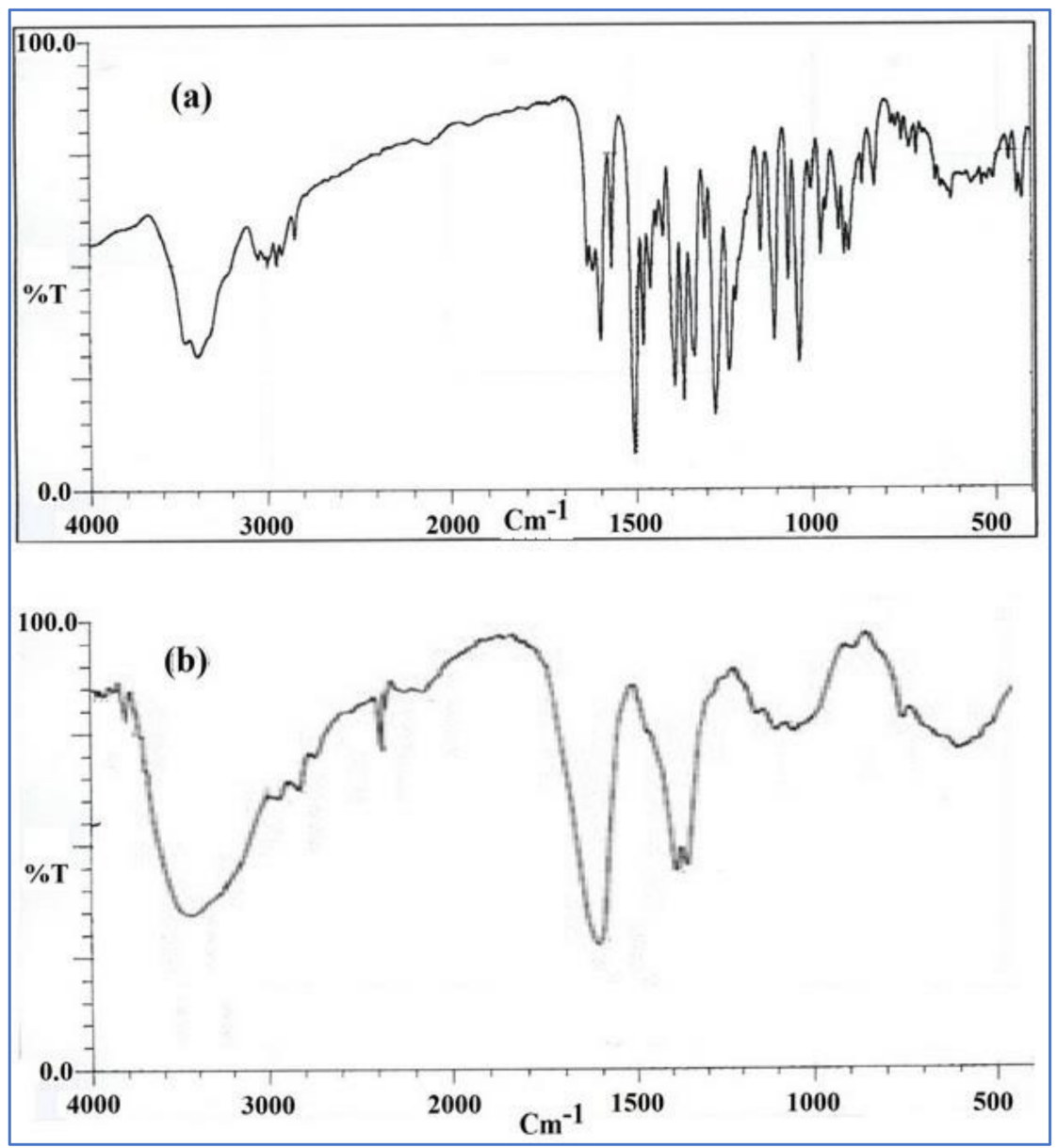

Figure 3. FTIR spectra of (a) pure BBR and (b) BNPs. 


\subsection{Nanoparticle Morphology}

The combination of SEM and TEM is the direct method to evaluate the size, surface morphology and vesicular structure of NPs [19]. The TEM image of BNPs showed the discrete particles with a solid, dense structure, with a size ranging from 170 to $303 \mathrm{~nm}$, which was in agreement with the results obtained from the PS measurement by the DLS technique (Figure 4a). The surface morphology of BNPs was analyzed by SEM techniques, and the SEM image results showed an irregularly smooth and rough surface (Figure $4 b$ ).

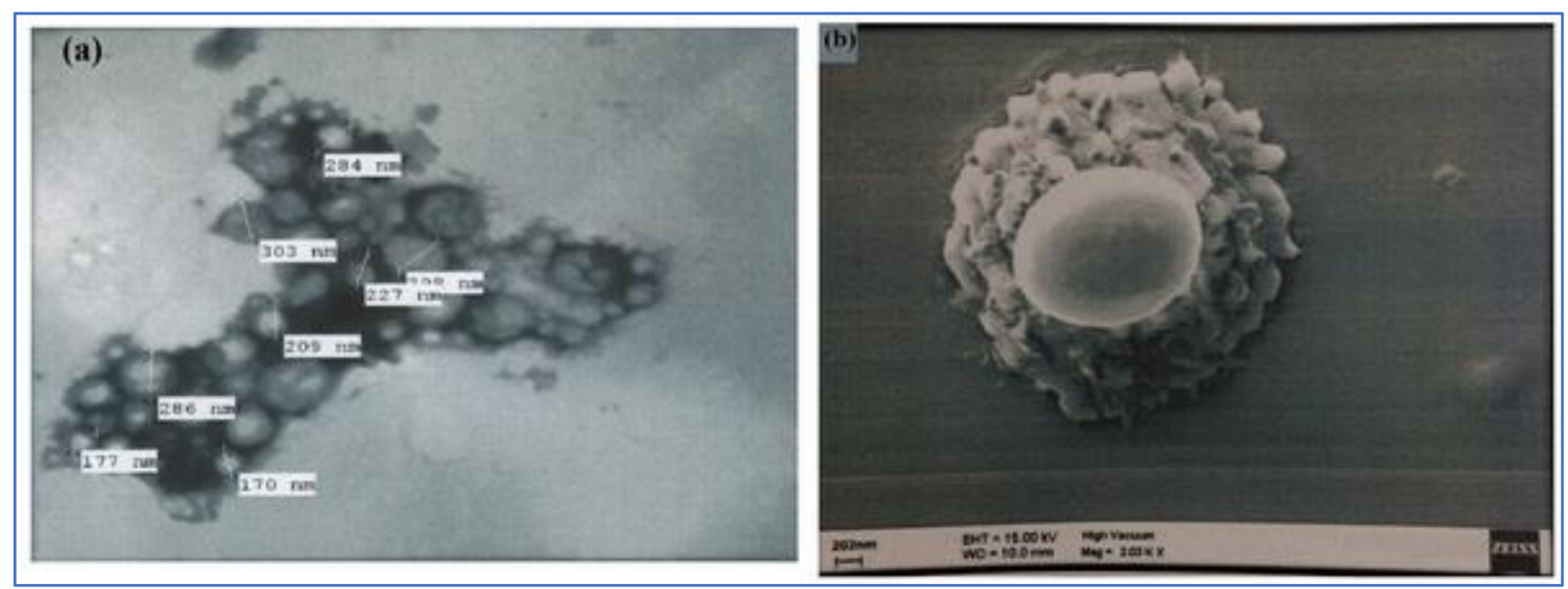

Figure 4. (a) TEM image of BNPs (b) SEM image of BNPs.

\subsection{In Vitro Drug-Release Kinetics}

The release study was conducted on lyophilized BNPs and was compared to that of free BBR suspension. It was observed that $100 \%$ of the free BBR was released in about $4 \mathrm{~h}$, whereas optimized BNP formulation showed an initial burst release of $10.49 \pm 1.5 \%$, followed by a continuous and controlled release of $75.06 \pm 4.2 \%$ of BBR in $24 \mathrm{~h}$ from BNPs (Figure 5). This initial release from BNPs could be most probably because of the presence of the drug on the surface of NPs or due to the diffusion of the drug from the pores, which are nearer to the surface of NPs [28]. Since NPs have a larger surface-area-to-volume ratio, such geometry is also favored for fast release [29].

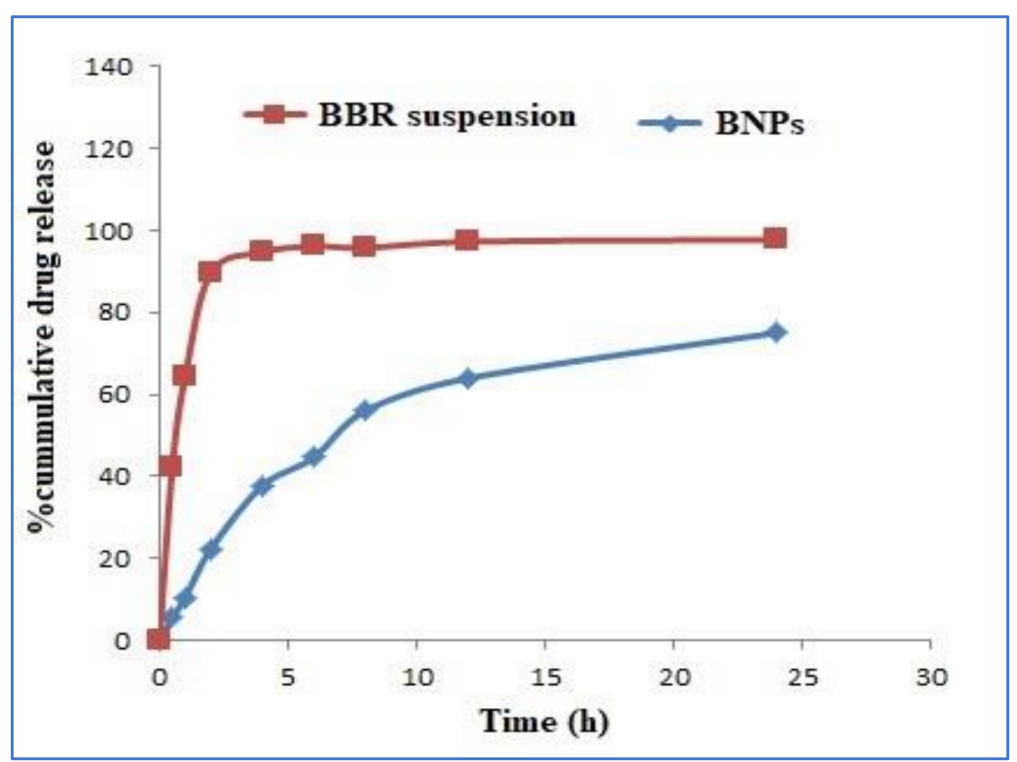

Figure 5. Cumulative release of BBR from BBR suspension and BNPs. 
To determine the release order, the in vitro release profile of the BNPs was kinetically analyzed. In the various release-kinetic models, such as zero-order, first-order, Higuchi, Hixcon-Crowell, and Korsmeyer-Peppas models, the data of the drug released were used, and the best-fit model was selected based on the value of regression coefficient $\left(R^{2}\right)$ [29]. It can be seen in Table 3 that BBR from BNPs follows the Higuchi release, for which a maximum $R^{2}$ value of 0.9636 was observed, which revealed the drug release from a matrixdiffusion-controlled system. The Korsmeyer-Peppas semi-empirical model was used to analyze the drug-release behavior from BNPs formulation on the linear proportion of the curve. The exponent constant $(n)$ was found at 0.39 , which suggested an anomalous Fickian-diffusion release, which reveals the drug release could be by both diffusion and erosion mechanisms.

Table 3. Regression coefficient $\left(R^{2}\right)$, release exponent (n) from the kinetic equations.

\begin{tabular}{ccccccccc}
\hline \multirow{2}{*}{ Formulation } & \multicolumn{2}{c}{ Zero-Order } & \multicolumn{2}{c}{ First Order } & \multicolumn{2}{c}{ Higuchi } & \multicolumn{3}{c}{ Korsmeyer-Peppas } \\
\cline { 2 - 9 } & $\mathbf{R}^{\mathbf{2}}$ & $\mathbf{k}$ & $\mathbf{R}^{\mathbf{2}}$ & $\mathbf{k}$ & $\mathbf{R}^{\mathbf{2}}$ & $\mathbf{k}$ & $\mathbf{n}$ & $\mathbf{R}^{\mathbf{2}}$ \\
\hline BNPs & 0.8050 & 3.113 & 0.9232 & 0.0258 & 0.9636 & 17.290 & 0.3907 & 0.9554 \\
BBR suspension & 0.283 & 2.372 & 0.4017 & 0.0535 & 0.5667 & 17.045 & 0.2401 & 0.6767 \\
\hline
\end{tabular}

\subsection{Stability Studies}

The optimized formulation of BNPs was evaluated for physical appearance, size analysis, drug content, and total \%drug release at various time intervals. It was observed that the BNP formulation showed no significant change in physical appearance, a very small change in mean PS, which was possibly due to aggregation of NPs over a period [11], and very little or no change in drug content in the three-month study period (Table 4). At both temperature conditions, i.e., $5 \pm 2{ }^{\circ} \mathrm{C}$ and $25 \pm 2{ }^{\circ} \mathrm{C}$, no significant changes were seen in the total BBR released from BNPs.

Table 4. Stability data.

\begin{tabular}{cccc}
\hline \multicolumn{4}{c}{ BNPs Formulation $\left(\mathbf{5} \pm \mathbf{2}{ }^{\circ} \mathbf{C}\right)$} \\
\hline Time (Days) & $\begin{array}{c}\text { Change in Physical } \\
\text { Appearance }\end{array}$ & Particle Size \pm SD $\mathbf{~ m ~}$ & Drug Content \\
\hline 0 & No & $202.2 \pm 1.60$ & $85.54 \pm 2.10$ \\
30 & No & $203.5 \pm 1.56$ & $84.89 \pm 1.46$ \\
60 & No & $203.9 \pm 3.21$ & $83.76 \pm 1.67$ \\
90 & No & $205.9 \pm 1.95$ & $83.23 \pm 1.69$ \\
& BNPs formulation $\left(\mathbf{2 5} \pm \mathbf{2}^{\circ} \mathbf{C}\right)$ & \\
0 & No & $202.2 \pm 1.60$ & $85.54 \pm 2.10$ \\
60 & No & $203.7 \pm 2.32$ & $84.23 \pm 1.26$ \\
90 & No & $205.6 \pm 3.29$ & $83.53 \pm 1.37$ \\
& No & $206.4 \pm 3.11$ & $82.98 \pm 1.83$ \\
\hline
\end{tabular}

\subsection{Ex Vivo Gut-Permeation Study}

Figure 6 represents the intestinal permeation profiles of BBR suspension and BNPs. It is evident from the graph that the permeation of BBR is enhanced 1.95-fold higher in BNPs than in BBR suspension. The enhancement of BBR permeation in BNPs could be most probably due to the inhibition of $P$-gp's expression on the gut lumen by the known $P$-gp inhibitors, i.e., chitosan and alginate [30]. The enhancement in permeation can also be attributed to the entrapment of BBR in nanoparticles, which otherwise is eliminated from the body, having only 5\% bioavailability [31]. Similarly, NPs themselves have a small size and also play an important role in enhancing permeation through the intestine [32-34]. 


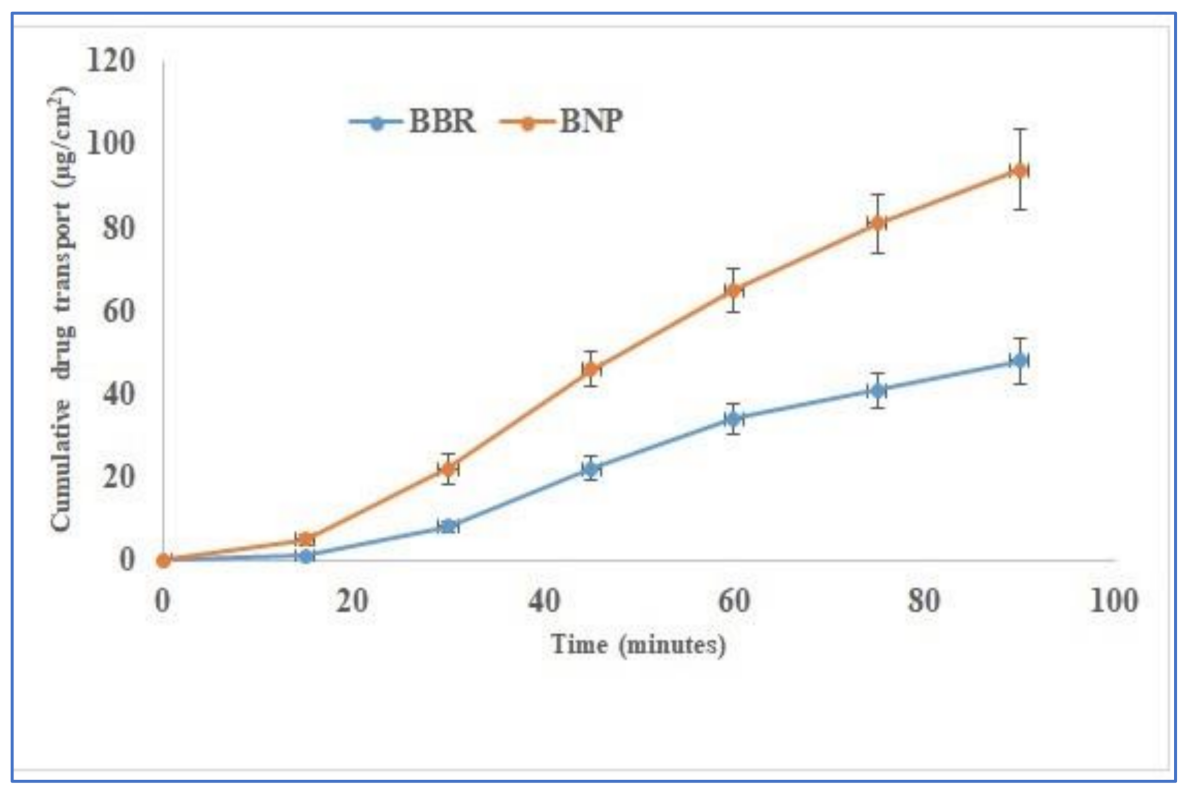

Figure 6. Ex vivo gut-permeation study showing comparative cumulative drug transport.

\subsection{Pharmacokinetic Studies}

The in vivo performance of the BBR suspension and BNPs was assessed for their pharmacokinetic performance for further verification of the above experiments and proof of concept. Pharmacokinetic analysis was carried out using the non-compartmental (modelindependent) method $[18,19]$. Figure 7 shows the plasma concentration-time profiles of BBR from BBR suspension and BNPs after oral administration, while Table 5 shows the various pharmacokinetic parameters.

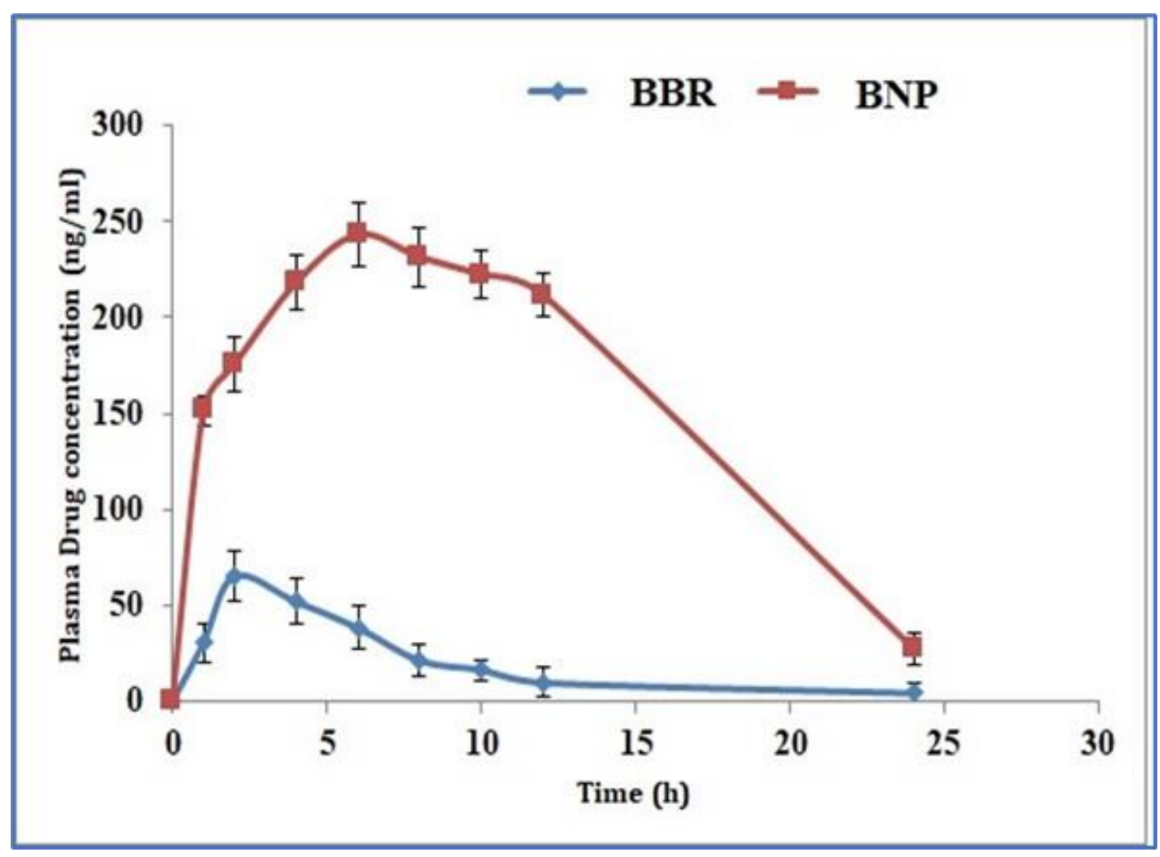

Figure 7. In vivo pharmacokinetics profile of BBR after oral administration of BBR suspension and BNPs (mean $\pm \mathrm{SD}, n=6$ ). 
Table 5. Pharmacokinetic parameters.

\begin{tabular}{ccc}
\hline Pharmacokinetic Parameters & BBR Suspension & BNPs \\
\hline $\mathrm{C}_{\max }(\mathrm{ng} / \mathrm{mL})$ & $67.54 \pm 3.90$ & $230.57 \pm 8.30$ \\
$\mathrm{~T}_{\max }(\mathrm{h})$ & $2.00 \pm 0.12$ & $6.00 \pm 0.02$ \\
$\mathrm{AUC}_{0-24}(\mathrm{ng} \cdot \mathrm{h} / \mathrm{mL})$ & $910.87 \pm 28.30$ & $3758.14 \pm 199.89$ \\
$\mathrm{Kel}\left(\mathrm{h}^{-1}\right)$ & $0.12 \pm 0.01$ & $0.08 \pm 0.003$ \\
$\mathrm{t}_{1 / 2}(\mathrm{~h})$ & $5.42 \pm 0.99$ & $9.04 \pm 0.17$ \\
Bioavailability enhancement $(\mathrm{F})$ & - & 4.13 \\
\hline
\end{tabular}

It was found that the $\mathrm{C}_{\max }$ of BBR from BNPs $(230.57 \pm 8.30 \mathrm{ng} / \mathrm{mL})$ significantly increased by 3.41-fold $(p<0.05)$ as compared to BBR suspension $(67.54 \pm 3.90 \mathrm{ng} / \mathrm{mL})$. This significant increase in $\mathrm{C}_{\max }$ value may be due to the $P$-gp inhibition by alginate and chitosan polymers, which are present in BNPs that enhance BBR absorption from the enterocytes. The $T_{\max }$ value of BNPs was also found to be significantly higher than BBR suspension, which may be due to sustained release of BBR from BNPs.

The areas under the curve $\left(\mathrm{AUC}_{0-24}\right)$ that denote the extent of absorption for $\mathrm{BBR}$ suspension and BNPs were found $910.87 \pm 28.30 \mathrm{ng} \cdot \mathrm{h} / \mathrm{mL}$ and $3758.14 \pm 199.89 \mathrm{ng} \cdot \mathrm{h} / \mathrm{mL}$, respectively. This means that the BBR absorption from BNPs was significantly higher than BBR suspension $(p<0.05)$. The pharmacokinetic data revealed that the BNPs have better oral bioavailability, as compared to BBR suspension, by a factor of 4.13 . This could be due to the $P$-gp inhibition by chitosan and alginate, which can prevent the drug efflux from the enterocytes [30]. Hence, these findings also support the gut-permeation study. The previously published work showed that the bioavailability of BBR in anhydrous reverse micelle and polymer-lipid hybrid nanoparticles was enhanced 2.4-fold and 3.34-fold only compared to BBR solutions $[35,36]$. Hence, our BNP formulations have good absorption efficiency and can enhance the medical performance of BBR as compared to existing formulations.

\section{Conclusions}

The oral formulation of poorly bioavailable drugs is challenging and needs further investigation. Developing an oral formulation for drugs like BBR with bioavailability $<5 \%$ is very challenging for pharmaceutical scientists. BNPs were successfully prepared by the ionic gelation method using P-gp inhibitors chitosan and sodium alginate, which could minimize the efflux of the drug out of the enterocytes. This also facilitated the entry of the drug through the intestinal cells. The size of the optimized BNPs was found to be $202.2 \pm 4.9 \mathrm{~nm}$, with $0.211 \pm 0.02$ of PDI. FTIR and DSC analyses suggested the there is no chemical interaction between BBR and the major excipients (chitosan and alginate) of the formulation. The SEM and TEM images of optimized BNPs showed smooth, spherical NPs with well-defined boundaries. The drug release from BNPs was found to show a burst release followed by a slow release, and it follows an anomalous Fickian-diffusion release, which reveals the drug release could be by both diffusion and erosion mechanisms. In addition, the in vivo pharmacokinetic analysis showed that the BNPs increased 4.10fold in improving the BBR oral bioavailability. Hence, this developed formulation with amalgamation benefit of the nano-sized system and polymeric $P$-gp modulator was found to be a good replacement for conventional oral formulation with a decrease dose and frequency of dosing.

Author Contributions: K.K.: supervision and conceptualization, A.M.: conceptualization, investigation, writing-original draft preparation, R.M.: methodology, data curation; S.A.: supervision and investigation; M.S.A.: writing-reviewing and editing; A.A.: funding, reviewing and editing; M.A.B.: formal analysis and editing; M.J.A.: funding, software, validation and editing. All authors have read and agreed to the published version of the manuscript.

Funding: The authors are acknowledge the Taif University researchers supporting project number (TURSP-2020/124), Taif University, Taif, Saudi Arabia for APC funding. 
Institutional Review Board Statement: The authors received animal ethical approval from the IAEC, Jamia Hamdard, New Delhi, India for in vivo pharmacokinetic study in Wistar rats with protocol no. 173/CPCSEA/2012. The study was conducted according to the guidelines of the Declaration of Helsinki.

Informed Consent Statement: The authors received animal ethical approval from the IAEC, Jamia Hamdard, New Delhi, India for in vivo pharmacokinetic study in Wistar rats with protocol no. 173/CPCSEA/2012.

Data Availability Statement: All data generated or analyzed during this study are included in this published article.

Acknowledgments: The authors acknowledge the Taif University researchers supporting project number (TURSP-2020/124), Taif University, Taif, Saudi Arabia. The authors are thankful to the Central Instrumental Facility (CIF) Laboratory, Jamia Hamdard, New Delhi, India for their assistance in carrying out the present research work. The authors gratefully acknowledge the Northern Border University, Saudi Arabia for providing online digital library facilities to help in manuscript preparation.

Conflicts of Interest: The authors report no conflicts of interest.

\section{References}

1. Ortiz, L.M.G.; Lombardi, P.; Tillhon, M.; Scovassi, A.I. Berberine, an Epiphany Against Cancer. Molecules 2014, 19, 12349-12367. [CrossRef] [PubMed]

2. Leng, S.-H.; Lu, F.-E.; Xu, L.-J. Therapeutic effects of berberine in impaired glucose tolerance rats and its influence on insulin secretion. Acta Pharmacol. Sin. 2004, 25, 496-502. [PubMed]

3. Tillhon, M.; Ortiz, L.M.G.; Lombardi, P.; Scovassi, A.I. Berberine: New perspectives for old remedies. Biochem. Pharmacol. 2012, 84, 1260-1267. [CrossRef]

4. Mujtaba, A.; Akhter, H.; Alam, S.; Ali, M.D.; Hussain, A. An updated review on therapeutic potential and recent advances in drug delivery of Berberine: Current status and future prospect. Curr. Pharm. Biotechnol. 2021, 22, 1. [CrossRef]

5. Liu, Y.-T.; Hao, H.-P.; Xie, H.-G.; Lai, L.; Wang, Q.; Liu, C.-X.; Wang, G.-J. Extensive Intestinal First-Pass Elimination and Predominant Hepatic Distribution of Berberine Explain Its Low Plasma Levels in Rats. Drug Metab. Dispos. 2010, 38, 1779-1784. [CrossRef]

6. Chen, W.; Miao, Y.-Q.; Fan, D.-J.; Yang, S.-S.; Lin, X.; Meng, L.-K.; Tang, X. Bioavailability Study of Berberine and the Enhancing Effects of TPGS on Intestinal Absorption in Rats. AAPS PharmSciTech 2011, 12, 705-711. [CrossRef]

7. Liu, C.-S.; Zheng, Y.-R.; Zhang, Y.-F.; Long, X.-Y. Research progress on berberine with a special focus on its oral bioavailability. Fitoterapia 2016, 109, 274-282. [CrossRef] [PubMed]

8. $\quad$ Feng, R.; Shou, J.-W.; Zhao, Z.-X.; He, C.-Y.; Ma, C.; Huang, M.; Fu, J.; Tan, X.-S.; Li, X.-Y.; Wen, B.-Y.; et al. Transforming berberine into its intestine-absorbable form by the gut microbiota. Sci. Rep. 2015, 5, 12155. [CrossRef] [PubMed]

9. Zhang, Y.; Cui, Y.-L.; Gao, L.-N.; Jiang, H.-L. Effects of $\beta$-cyclodextrin on the intestinal absorption of berberine hydrochloride, a P-glycoprotein substrate. Int. J. Biol. Macromol. 2013, 59, 363-371. [CrossRef]

10. Ahmad, M.Z.; Ahmad, J.R.; Alasmary, M.Y.; Akhter, H.; Abdel-Wahab, B.A.; Warsi, M.H.; Haque, A. Rizwanullah Progress in nanomedicine-based drug delivery in designing of chitosan nanoparticles for cancer therapy. Int. J. Polym. Mater. 2021, 1-22. [CrossRef]

11. Akhter, H.; Kumar, S.; Nomani, S. Sonication tailored enhance cytotoxicity of naringenin nanoparticle in pancreatic cancer: Design, optimization, and in vitro studies. Drug Dev. Ind. Pharm. 2020, 46, 659-672. [PubMed]

12. Loquercio, A.; Castell-Perez, E.; Gomes, C.; Moreira, R.G. Preparation of Chitosan-Alginate Nanoparticles forTranscinnamaldehyde Entrapment. J. Food Sci. 2015, 80, N2305-N2315. [CrossRef] [PubMed]

13. Katuwavila, N.P.; Perera, A.D.L.C.; Samarakoon, S.R.; Soysa, P.; Karunaratne, V.; Amaratunga, G.A.J.; Karunaratne, D.N. Chitosan-alginate nanoparticle system efficiently delivers doxorubicin to MCF-7 Cells. J. Nanomater. 2016, 2016, 3178904. [CrossRef]

14. Li, Y.-J.; Hu, X.-B.; Lu, X.-L.; Liao, D.-H.; Tang, T.-T.; Wu, J.-Y.; Xiang, D.-X. Nanoemulsion-based delivery system for enhanced oral bioavailability and Caco-2 cell monolayers permeability of berberine hydrochloride. Drug Deliv. 2017, 24, 1868-1873. [CrossRef] [PubMed]

15. Yadav, K.; Sawant, K.K. Formulation Optimization of Etoposide Loaded PLGA Nanoparticles by Double Factorial Design and their Evaluation. Curr. Drug Deliv. 2010, 7, 51-64. [CrossRef] [PubMed]

16. Mujtaba, M.A.; Hassan, K.A.M.; Imran, M. Chitosan-alginate nanoparticles as a novel drug delivery system for rutin. Int. J. Adv. Biotechnol. Res. 2018, 9, 1895-1903.

17. Akhter, M.H.; Ahmad, A.; Ali, J. Formulation and Development of CoQ10-Loaded s-SNEDDS for Enhancement of Oral Bioavailability. J. Pharm. Innov. 2014, 9, 121-131. [CrossRef] 
18. Singh, A.; Neupane, Y.R.; Shafi, S.; Mangla, B.; Kohli, K. PEGylated liposomes as an emerging therapeutic platform for oral nanomedicine in cancer therapy: In vitro and in vivo assessment. J. Mol. Liq. 2020, 303, 112649.

19. Soni, K.; Mujtaba, A.; Kohli, K. Lipid drug conjugate nanoparticle as a potential nanocarrier for the oral delivery of pemetrexed diacid: Formulation design, characterization, ex vivo, and in vivo assessment. Int. J. Biol. Macromol. 2017, 103, 139-151. [CrossRef] [PubMed]

20. Mujtaba, M.A.; Alotaibi, N.M. Chitosan-sodium alginate nanoparticle as a promising approach for oral delivery of rosuvastatin calcium: Formulation, optimization and in vitro characterization. J. Pharm. Res. Int. 2020, 32, 50-56. [CrossRef]

21. Sorasitthiyanukarn, F.N.; Muangnoi, C.; Na Bhuket, P.R.; Rojsitthisak, P.; Rojsitthisak, P. Chitosan/alginate nanoparticles as a promising approach for oral delivery of curcumin diglutaric acid for cancer treatment. Mater. Sci. Eng. C 2018, 93, 178-190. [CrossRef] [PubMed]

22. Mujtaba, A.; Ali, M.; Kohli, K. Statistical optimization and characterization of $\mathrm{pH}$-independent extended-release drug delivery of cefpodoxime proxetil using Box-Behnken design. Chem. Eng. Res. Des. 2014, 92, 156-165. [CrossRef]

23. Thai, H.; Nguyen, C.T.; Thach, L.T.; Tran, M.T.; Mai, H.D.; Nguyen, T.T.T.; Le, G.D.; Van Can, M.; Tran, L.D.; Bach, G.L.; et al. Characterization of chitosan/alginate/lovastatin nanoparticles and investigation of their toxic effects in vitro and in vivo. Sci. Rep. 2020, 10, 909-915. [CrossRef] [PubMed]

24. Douglas, K.L.; Tabrizian, M. Effect of experimental parameters on the formation of alginate-chitosan nanoparticles and evaluation of their potential application as DNA carrier. J. Biomater. Sci. Polym. Ed. 2005, 16, 43-56. [CrossRef]

25. Mujtaba, A.; Ali, M.; Kohli, K. Formulation of extended release cefpodoxime proxetil chitosan-alginate beads using quality by design approach. Int. J. Biol. Macromol. 2014, 69, 420-429. [CrossRef]

26. Yeo, Y.; Park, K. Control of encapsulation efficiency and initial burst in polymeric microparticle systems. Arch. Pharmacal Res. 2004, 27, 1.

27. Battu, S.K.; Repka, M.A.; Maddineni, S.; Chittiboyina, A.; Avery, M.; Majumdar, S. Physicochemical Characterization of Berberine Chloride: A Perspective in the Development of a Solution Dosage Form for Oral Delivery. AAPS PharmSciTech. 2010, 11, 1466-1475. [CrossRef] [PubMed]

28. Khuroo, T.; Verma, D.; Talegaonkar, S.; Padhi, S.; Panda, A.K.; Iqbal, Z. Topotecan-tamoxifen duple PLGA polymeric nanoparticles: Investigation of in vitro, in vivo and cellular uptake potential. Int. J. Pharm. 2014, 473, 384-394. [CrossRef] [PubMed]

29. Mujtaba, A.; Kohli, K. In vitro/in vivo evaluation of HPMC/alginate based extended-release matrix tablets of cefpodoxime proxetil. Int. J. Biol. Macromol. 2016, 89, 434-441. [PubMed]

30. Werle, M. Natural and Synthetic Polymers as Inhibitors of Drug Efflux Pumps. Pharm. Res. 2008, 25, 500-511. [CrossRef] [PubMed]

31. Vuddanda, P.R.; Chakraborty, S.; Singh, S. Berberine: A potential phytochemical with multispectrum therapeutic activities. Expert Opin. Investig. Drugs 2010, 19, 1297-1307. [PubMed]

32. Liu, W.; Pan, H.; Zhang, C.; Zhao, L.; Zhao, R.; Zhu, Y.; Pan, W. Developments in Methods for Measuring the Intestinal Absorption of Nanoparticle-Bound Drugs. Int. J. Mol. Sci. 2016, 17, 1171. [CrossRef] [PubMed]

33. Blanco, E.; Shen, H.; Ferrari, M. Principles of nanoparticle design for overcoming biological barriers to drug delivery. Nat. Biotechnol. 2015, 33, 941-951. [CrossRef] [PubMed]

34. Singh, A.; Ahmad, I.; Akhter, S.; Jain, G.K.; Iqbal, Z.; Talegaonkar, S.; Ahmad, F.J. Nanocarrier based formulation of Thymoquinone improves oral delivery: Stability assessment, in vitro and in vivo studies. Colloids Surf. B Biointerfaces 2013, 102, 822-832. [PubMed]

35. Wang, T.; Wang, N.; Song, H.; Xi, X.; Wang, J.; Hao, A.; Li, T. Preparation of an anhydrous reverse micelle delivery system to enhance oral bioavailability and anti-diabetic efficacy of berberine. Eur. J. Pharm. Sci. 2011, 44, 127-135. [CrossRef] [PubMed]

36. Yu, F.; Ao, M.; Zheng, X.; Li, N.; Xia, J.; Li, Y.; Li, D.; Hou, Z.; Qi, Z.; Chen, X.D. PEG-lipid-PLGA hybrid nanoparticles loaded with berberine-phospholipid complex to facilitate the oral delivery efficiency. Drug Deliv. 2017, 24, 825-833. [CrossRef] 